\title{
RF Applications of Superconducting Tunneling Devices
}

\section{Pedersen, Niels Falsig}

Published in:

13th European Microwave Conference

Link to article, DOI:

10.1109/EUMA.1983.333213

Publication date:

1983

Document Version

Publisher's PDF, also known as Version of record

Link back to DTU Orbit

Citation (APA):

Pedersen, N. F. (1983). RF Applications of Superconducting Tunneling Devices. In 13th European Microwave Conference (pp. 88-96). IEEE. https://doi.org/10.1109/EUMA.1983.333213

\section{General rights}

Copyright and moral rights for the publications made accessible in the public portal are retained by the authors and/or other copyright owners and it is a condition of accessing publications that users recognise and abide by the legal requirements associated with these rights.

- Users may download and print one copy of any publication from the public portal for the purpose of private study or research.

- You may not further distribute the material or use it for any profit-making activity or commercial gain

- You may freely distribute the URL identifying the publication in the public portal

If you believe that this document breaches copyright please contact us providing details, and we will remove access to the work immediately and investigate your claim 
N. Falsig Pedersen

\section{INTRODUCTION}

In recent years a lot of interest has been focused on the Josephson junction as a microwave device in the millimeter and submillimeter range. The primary reason for this interest is that it has been verified experimentally that the Josephson junction makes the receiver with the lowest noise temperature in a large part of the microwave spectrum. Already now radio astronomy receivers with a Josephson junction as the sensitive element have been tested sucessfully and total receiver noise temperatures as low as $150 \mathrm{~K}$ have been realized in the 40-120 $\mathrm{GHz}$ range. Although the interest is now focused largely on the so-called SIS mixer, the Josephson junction being a highly nonlinear device may be used also as a parametric amplifier. For both mixers and parametric amplifiers, modes of operation exist, in which the pump (or local oscillator) is inherent in the junction; alternatively, another Josephson junction may be used as a microwave generator. That unique feature becomes attractive in the higher end of the microwave spectrum where sources are typically bulky and expensive. The development of practically usable Josephson microwave elements is still being pursued actively around the world. A noticeable trend now is that this research is increasingly being transferred from the physics laboratories to the microwave community; the progress that has taken place has also benefitted much from large research projects aimed at developping a new generation of computers based on Josephson junctions. Several good recent reviews of the field exists $1,2,3,4,5,6$ and may serve as an introduction for those who wish more information.

\section{THE JOSEPHSON JUNCTION}

Superconductivity, which is fundamental to the Josephson junction, may be described as the total loss of electrical resistance and the exclusion of magnetic flux in some metals below a transition temperature $\mathrm{T}_{\mathrm{C}}$. Typical materials used are lead, niobium, and alloys with $\mathrm{T}_{\mathrm{C}}$ 's of order $5-10 \mathrm{~K}$. A Josephson junction consists typically of two thin films made of such a material and separated by a thin (10 - $50 \AA)$ insulating barrier. Fig. 1 shows schematically such a structure, and Fig. 2 shows a typical current voltage characteristic.

The IV characteristic has two branches which are conceptually separable in that the current flowing at zero voltage (the supercurrent)originates from tunneling cooper pairs, consisting of two electrons which are coupled together. These Cooper pairs

Physics Laboratory I, Technical University of Denmark, DK 2800 Lyngby, Denmark. 
Fig. 1. Schematic view of a Josephson junction consisting of two superconducting films separated by an insulating barrier.

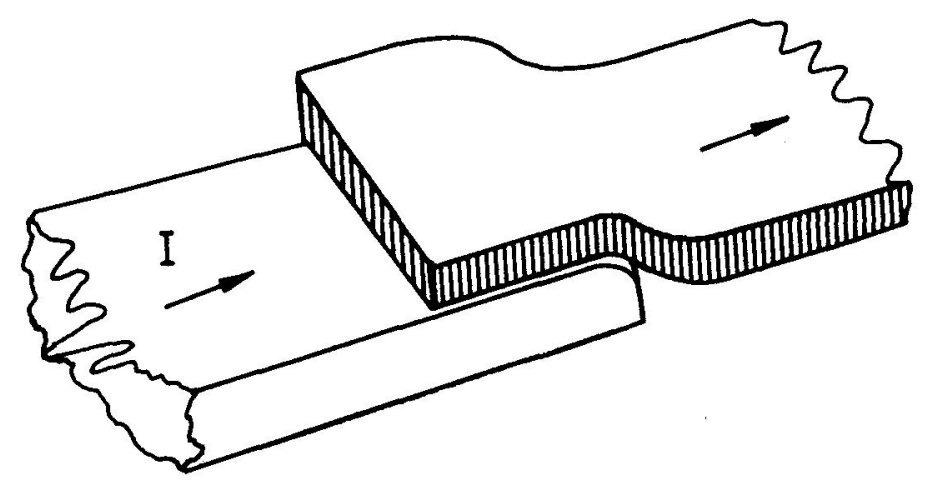

Fig. 2. Typical IVcharacteristic of a Josephson junction. Bias points $A$ and $B$ correspond to parametric amplifiers and $\mathrm{C}$ corresponds to a SIs mixer.

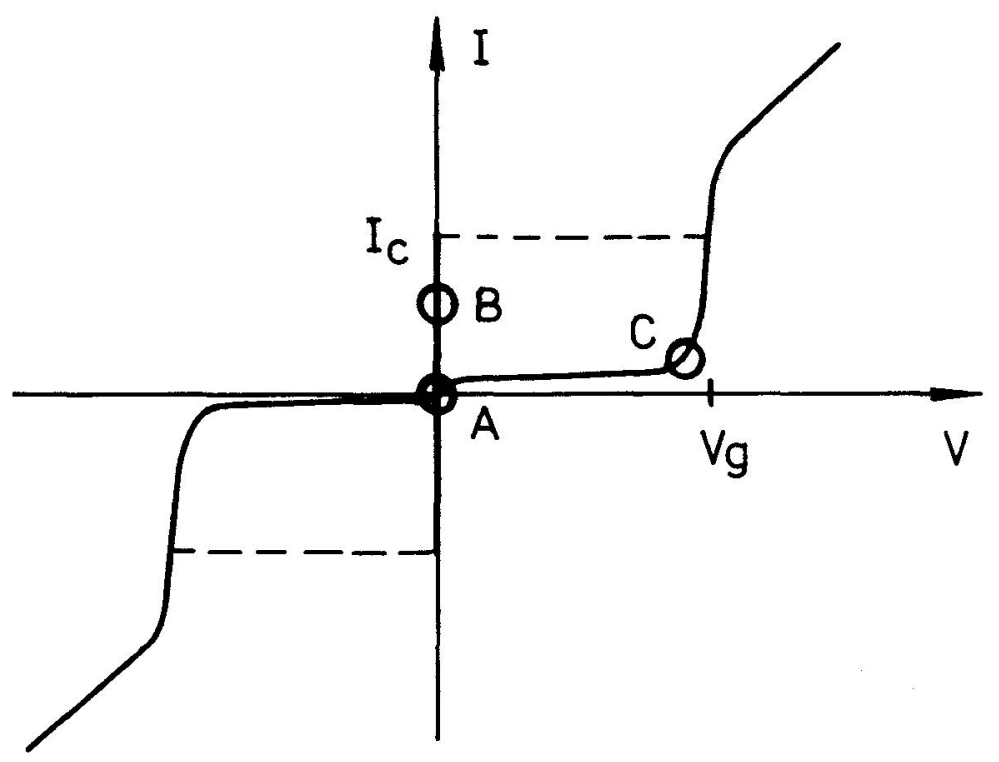

propagate without losses. The current flowing at finite voltages originates from unpaired electrons, the so-called quasiparticles. A prominent feature of this part of the IV curve is the threshold voltage $V_{g}$ (called the energy gap) below which the current is very low?

For voltages greater than zero but less than the energy gap also the Cooper pairs tunnel through the barrier; the amplitude of that current contribution (the supercurrent) is the same as at zero voltage, however, it is rapidly oscillating at a frequency of $484 \mathrm{MHz} / \mu \mathrm{V}$, and averages to zero. Since $\mathrm{V}_{\mathrm{g}}$ may be of order $3 \mathrm{mV}$ this oscillation frequency may be as high as $1500 \mathrm{GHz}$ indicating the frequency range of the non-linearities.

The above simplified discussion may be written mathematically in the following way

$$
C \cdot \frac{d V}{d t}+\frac{V}{R(V)}+I_{o} \sin \phi=I_{d c}
$$




$$
\frac{\mathrm{d} \phi}{\mathrm{d} t}=\frac{2 \mathrm{eV}}{\mathrm{h}}
$$

In Eq. (1) the first term is a capacitive current, the second is the quasiparticle current, and the third term is the Cooper pair current. The significance of $\phi$ is that it is the phase difference of the wave function of the cooper pairs on either side of the junction. The second Josephson equation relates the time rate of change of the phase difference to the voltage. The proportionality constant $2 \mathrm{e} / \mathrm{h}$ is $484 \mathrm{MHz} / \mu \mathrm{V}$ (e = electron charge, $\mathrm{h}=\mathrm{Planck}$ 's constant). Fig. 3 shows an equivalent diagram of the Josephson junction, in accordance with Equation 1.

Fig. 3. Equivalent diagram of a Josephson junction.

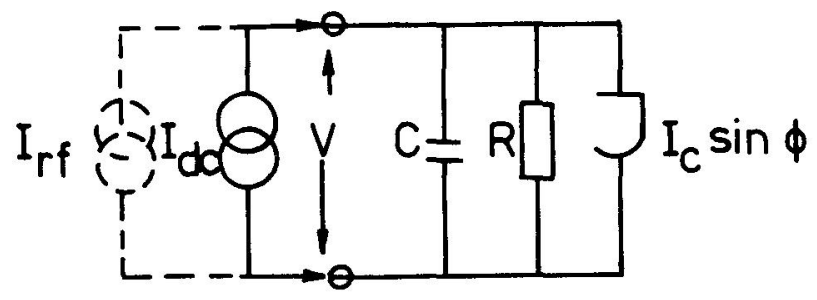

Eqs. 1 and 2 may be combined to a nonlinear second order differential equation in $\phi$; this differential equation has the same form as the pendulum equation or the phase-locked loop equation In the following we will consider some specific examples of rf applications of Josephson junctions.

\section{THE EXTERNALLY PUMPED PARAMETRIC AMPLIFIER}

The parametric amplifier employ the nonlinear properties of the supercurrent at zero voltage. Differentiating the supercurrent part, $I_{S}$, with respect to time we obtain

$$
\frac{d I_{S}}{d t}=\frac{d}{d t}\left(I_{0} \sin \phi\right)=I_{0} \cos \phi \cdot \frac{d \phi}{d t}=\frac{2 e I_{0}}{h} \cdot \cos \phi V
$$

The proportionality between $d I_{s} / d t$ and the voltage implies that the supercurrent at zero voltage is equivalent to a nonlinear inductor, the value of which is tunable by the dc bias current

$$
L=\frac{h}{2 e I_{0}} \cdot \frac{I}{\cos \phi}=\frac{K}{2 e I_{0} \sqrt{1-\left(\frac{I_{d c}}{I_{0}}\right)^{2}}}
$$

since from Eq. (I) at zero voltage $\sin \phi=I_{d c} / I_{0}$. Together with the capacitance $\mathrm{C}$ this inductance gives a resonance frequency 
$\omega_{p}=1 / \sqrt{\mathrm{LC}}$ - the socalled plasma resonance - which is typically in the range $10-300 \mathrm{GHz}$ depending on controllable fabrication parameters. If now an external (pump) rf current is applied (an additional term $I_{r f}$ sirwt appears on the right hand side of Eq. (1) the situation is rather similar to an externally pumped varactor diode parametric amplifier, except that here it is the non linear inductance being modulated at the pumping frequency, whereas in the varactor amplifier it is the non linear capacitance. Two schemes have been investigated both experimentally and theoretically. In one scheme the pump is at twice the signal frequency ( 3 photon amplifier) and in the other the signal, idler and pump frequencies are about equal. (4 photon amplifiers). In both cases the signal frequency is in the vicinity of the plasma resonance. Table I gives typical numbers for what has been obtained with Josephson junction parametric amplifiers.

Table I. Parametric amplifier results

\begin{tabular}{ccrcc}
\hline reference & $\begin{array}{c}\text { fsignal } \\
\text { GHz }\end{array}$ & $\begin{array}{r}\text { noise temp. } \\
\mathrm{K}\end{array}$ & $\begin{array}{c}\text { gain } \\
\mathrm{dB}\end{array}$ & mode \\
\hline 5 & 33 & 40 & 15 & 4 photon \\
6 & 36 & $<50$ & 11 & - " \\
7 & 10 & 30 & $>20$ & - " \\
8 & 35 & $\sim 50$ & $4-12$ & 3 photon \\
\hline
\end{tabular}

Although the numbers in table I appear very promising, there are problems with the Josephson junction parametric amplifier. One drawback is the low signal saturation level, typically of order $10^{-11} \mathrm{~W}$. Another is the low impedance levels. As the frequency is raised the current density must be increased in order to raise the plasma frequency. A high current density requires small junctions $(1 \sim 10 \mu \mathrm{m})$ which makes fabrication more difficult. Both these problems can be circumvented to some extent by using a series array of junctions. Another drawback is that the origin of the noise in Josephson junction parametric amplifiers is not fully understood, and may involve more complex noise contributions than traditional Johnson noise or shot noise.

A theoretically estimated upper frequency for the Josephson parametric amplifier is $300 \mathrm{GHz}$.

\section{THE INTERNALLY PUMPED PARAMETRIC AMPLIFIER}

Several groups have investigated the internally pumped Josephson parametric amplifier, in which the supercurrent oscillation at $484 \mathrm{GHz} / \mathrm{mv}$ (constant voltage bias) is used as the pump source. In particular a recent experiment at $10 \mathrm{GHz}$ done by the group in Gothenburg 9 showed gains of order $5 \mathrm{~dB}$ and noise temperatures below $25 \mathrm{~K}$. It is not yet known whether such results can be obtained at higher frequencies. 


\begin{tabular}{lccc} 
Table II & SIS mixer & results \\
\hline reference & $\begin{array}{c}\text { fsignal } \\
\mathrm{GHz}\end{array}$ & $\begin{array}{c}\text { mixer } \\
\text { noise temp. } \\
\mathrm{k}\end{array}$ & conversion $^{\left(\mathrm{L}_{\mathrm{SSB}}\right)^{-1}}$ \\
\hline 11 & & $\sim 10$ & 0.26 \\
12 & 9 & 10 & 2.7 \\
13 & 36 & 15 & 0.32 \\
14 & $35-50$ & 27 & 0.23 \\
15 & 55 & $5 \pm 94$ & 0.52 \\
16 & 70 & $20 \pm 90$ & 0.63 \\
17 & 74 & $70 \pm 40$ & 0.07 \\
18 & 115 & 60 & 0.2 \\
19 & 115 & $<300$ & \\
\hline
\end{tabular}

Table II shows some results for conversion efficiency and mixer noise temperature. In references 13 and 18 the SIS mixer was actually mounted in radio telescopes; in 13 total receiver noise temperatures between $140 \mathrm{~K}$ and $220 \mathrm{~K}$ were reported. In refs. 12, 15 and 16 it can safely be noticed that conversion efficiencies are larger than possible with classical mixing. (In other experiments a low conversion efficiency may be due to poor impedance matching). The work with using SIS mixers in radio telescopes are being actively pursued. Theoretical estimates of the upper frequency are in the $3-500 \mathrm{GHz}$ range.

\section{SUMMARY OF SUPERCONDUCTING TUNNEL JUNCTION DETECTORS}

There are other devices than mentioned here, for example the socalled Josephson mixer which is typically a superconducting point contact. This mixer is known to work at frequencies of order $1000 \mathrm{GHz}$ or even larger, however, the conversion efficiency and noise temperature are typically somewhat inferior to the SIS mixer. Fig. (5) shows a state of the art diagram of (SSB) noise temperatures for various high frequency detectors based on Josephson junctions. The quantum limit is shown as the full line.

\section{GENERATION OF ELECTROMAGNETIC RADIATION}

A unique feature of the Josephson junction is that when biased at a constant voltage $V_{O}$, the supercurrent oscillates with a frequency $\omega=2 \mathrm{eV}_{\mathrm{O}} / \mathrm{h}$ as discussed previously. This effect makes the basis for another potential application of the Josephson junction namely as a tunable source of high frequency radiation This application is not yet as mature as that of radiation detection, however, research is being continued in many laboratories 
The so-called SIS (superconductor-insulator-superconductor) mixer has made remarkable progress in recent years. It is quite different from most other Josephson junction elements, which employs the properties of the supercurrent. The SIS mixer uses a bias point corresponding to point C in Fig. (2), and may be described as working on the borderline between the classical and quantum limits. At high frequencies when the photon energy is larger than the singular region of the IV curve at the gap voltage $\mathrm{V}_{\mathrm{g}}$ (Fig. (2)) the quantum nature of the problem may even give rise to conversion gain.

Classically, the SIS mixer simply make use of the sharp nonlinearity of the IV curve (bias point C in Fig. (2)) and may accordingly be considered as a classical mixer; hence the curvature and slope in the bias point together with relevant input and output impedances determine the conversion efficiency, $\mathrm{IC}^{-1}$ from rf to if - frequency. Even with idealized and carefully designed circuits $L_{C}^{-}$can never be larger than 0.5 . The curvature parameter $\mathrm{S}=\mathrm{d}^{2} \mathrm{I} / \mathrm{dV}^{2} / \mathrm{dI} / \mathrm{dV}$ can be used to compare the conversion efficiencies of different devices. In a schottky barrier diode $\mathrm{S} \simeq 50 \mathrm{~V}^{-1}$ at room temperature. BY making the metal point a superconductor (Super Schottky diode) $S$ values of order $10^{4} \mathrm{~V}^{-1}$ have been obtained at low temperatures. The SIS junction at low temperatures may display an even stronger curvature.

However, even the very large s-value of a classical SIS mixer does not give an explanation of the observed conversion gain. A quantum picture is necessary. Photons can be absorbed or emitted by electrons during the tunneling process. This photon induced tunneling due to an applied local oscillator signalgives rise to structure around the energy gap as shown in Fig. (4). The average current at bias voltage $V$ is not only a function of $\mathrm{V}$ but also of $\mathrm{V}+\mathrm{nhf}_{\mathrm{LO}} / 2(\mathrm{n}=1,2, \ldots)$. A full quantum mechanical treatment was given by Tucker $10^{\circ}$, who showed that conversion gain may occur in the SIS mixer if the quantum effects are taken into account. A condition for the conversion gain to occur is that the quasiparticle corner is sharp on the scale of $\mathrm{hf}_{\mathrm{LO}} / \mathrm{e}$. This can be obtained by choosing a suitable (high) frequency, (low) temperature, and the right junction materials.

Fig. 4. SIS-mixer: IV curve without (full curve) and with (dashed curve) local oscillator power.

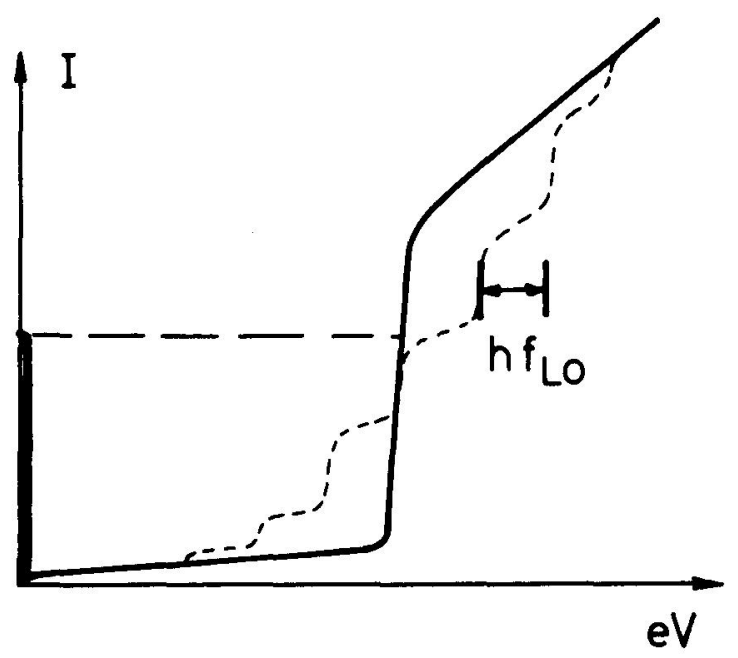


Fig. 5. SSB noise temperature of the various Josephson junction receiving devices. The full line indicates the quantum limit.

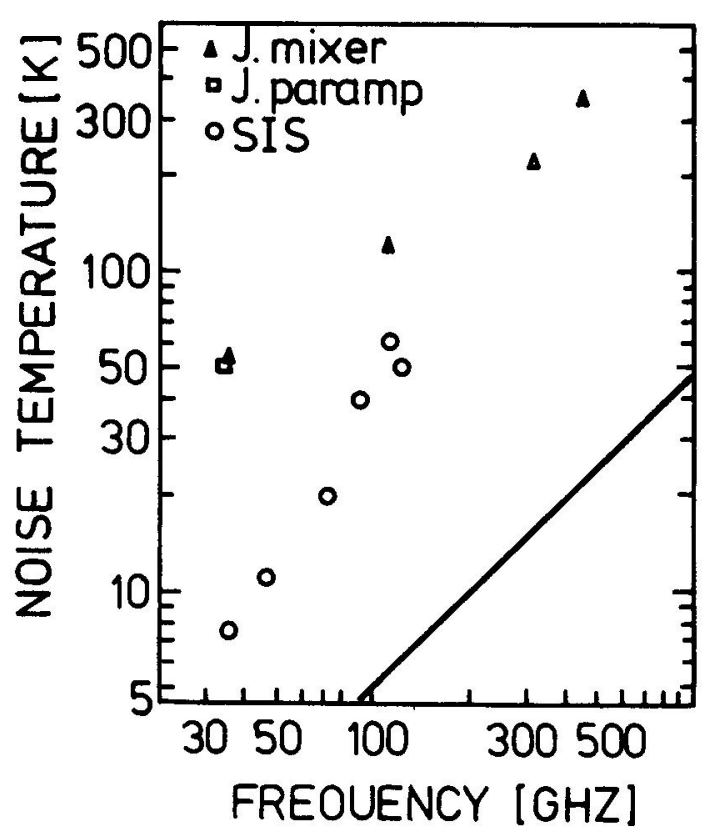

The applications that one can foresee is the use of a Josephson junction oscillator as the LO or pump source for SIS mixers or parametric amplifiers. The power requirement for such applications is in the range $10^{-8}-10^{-10}$ watt and probably rather narrow linewidths ( $\leqslant 1 \mathrm{MHz}$ ) will be needed.

In tunnel junctions radiation generation may for example be obtained by using a long junction in which solitons (a stable voltage pulse) bounce back and forth at the desired frequency of radiation. Those solitons give rise to so-called zero field steps in the IV curve as shown in Fig. (6). The power delivered to an external load may be maximized by increasing the current density of the junction, and by a careful design of the coupling circuit to the external world. A major problem here is that the characteristic impedance of this Josephson transmission line is low, typically less than $1 \Omega$. Nevertheless $10^{-10}$ watts at $10 \mathrm{GHz}$ is relatively easy to obtain without elaborate coupling circuits. Recently $5 \times 10^{-9}$ Watts delivered in a regular waveguide was obtained at $35 \mathrm{GHz}^{19}$. Also recently a remarkable experiment was performed in which the power was delivered into another low impedance junction on the substrate in order to circumvent the large impedance transformation. In this case powers as large as $10^{-6}$ watts at $100-400 \mathrm{GHz}$ was obtained. Only few measurements of the linewidths have been performed, however in one case linewidths between 3 and $100 \mathrm{kHz}$ at $10 \mathrm{GHz}$ was obtained in a temperature stabilized cryostat.

Another approach to enhance the power generation is to make an array of small coupled junctions. If the junctions act coherently the power will increase as the number of junctions squared. For an array of 100 junctions a power of $10^{-8} \mathrm{~W}$ was delivered in a $50 \Omega$ load and with a linewidth of $1 \mathrm{MHz}$ at $10 \mathrm{GHz}$.

Those array samples offer some advantages in tunability, however, 
Fig. 6. IV curve for a long Josephson junction. Circle indicates bias point when used as a soliton microwave generator.

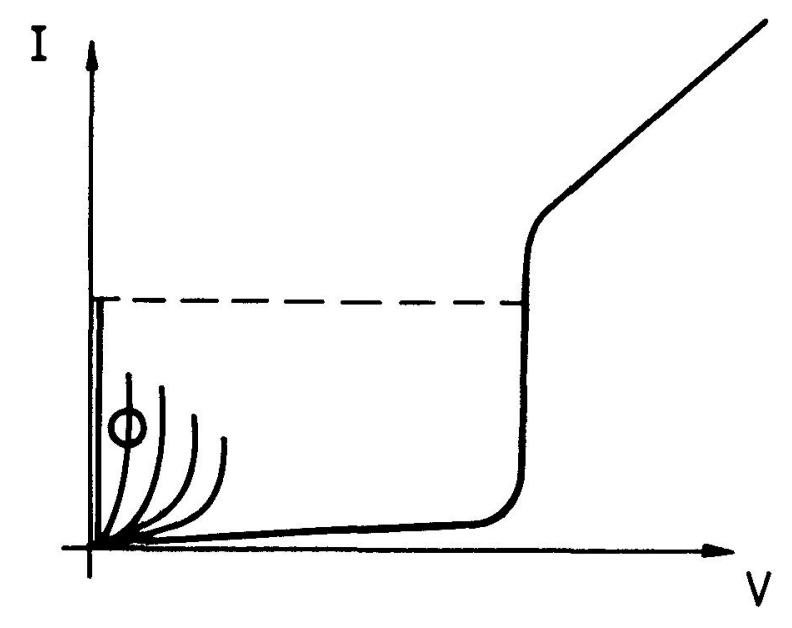

their fabrication require very elaborate photolithographic facilities to work with submicron dimensions.

\section{CONCLUSION}

The Josephson junction appears to be a microwave element that must be taken seriously in the future. It offers a great potential for making integrated microwave circuitry where antennas, filters, amplifiers, generators, and mixers are all part of the same substrate. Frequencies up to at least $500 \mathrm{GHz}$ appear feasible. Although a breakthrough was obtained with the development and subsequent practical use of the SIS mixer, promises are even larger than what has already been obtained. Applied research done - not so much by physicists - but by microwave engineers could prove to be very rewarding.

\section{REFERENCES}

1. R.Y. Chiao, IEEE Trans. Magn. MAG-15, 446, (1979).

2. N.F. Pedersen, in "SQUID '80" (H.D. Hahlbohm and H. Lübbig, eds.) W. de Gruyter, Berlin 1980, p. 739 .

3. P.L. Richards and T-M Shen, IEEE Trans. El. Devices ED-27, 1909, (1980).

4. T. Claeson, NATO Summer institute, Advances in Superconductivity, Erice 1982. In print (Plenum Press).

5. R.Y. Chiao and P.T. Parrish. J.Appl.Phys. 47, 2639, (1976).

6. Y. Taur and P.L. Richards. J.Appl.Phys. 48, 1321, (1977).

7. S. Wahlsten, S. Rudner and T. Claeson, J.Appl.Phys. 49 , 4248 , (1978).

8. J. Mygind, N.F. Pedersen, O.H. Soerensen, B. Dueholm and M.T. Levinsen, Appl.Phys.Lett. 35, 91, (1979). 
9. N. Calander, T. Claeson and S. Rudner, Appl.Phys.Lett. $\underline{39}$, 650, (1981).

10. J.R. Tucker, IEEE J. Quantum Electron, QE-15, 1234, (1979) Appl.Phys.Lett. 36, 477, (1980).

11. S. Rudner and T. Claeson, Appl.Phys.Lett. 34, 713, (1979).

12. A.D. Smith, W.R. McGrath, P.L. Richards, H. van Kempen, D. Prober and P. Santhanam, Physica 108B, 1367, (1981).

13. L. Olsson, S. Rudner, E. Kollberg and C.O. Lindström, to be published.

14. A.C. Callegari and R.A. Buhrman, J.Appl.Phys. 53, 823, (1982)

15. H.J. Hartfuss and K.H. Gundlach, Int. J. of Infrared and Millimeter Waves $\underline{2}$, No. 5, (1981).

16. S. Rudner, M.J. Feldman, E. Kollberg and T. Claeson, IEEE Trans. Magn. MAG-17, 690, (1981); J.Appl.Phys. 52, 6366, (1981).

17. A.R. Kerr, S.-K. Pan, M.J. Feldman and A. Davidson, Physica 108B, 1369, (1981).

18. T.G. Phillips, D.P. Woody, G.J. Dolan, R.E. Miller and R.A. Linke, IEEE Trans. Magn. MAG-17, 684, (1981).

19. G.K. Hohenwarter, (Private communication).

20. T. Nagatsuma, K. Enpuku, F. Irie and K. Yoshida, Preprint (1982). 